\title{
In memory of Yurii Petrovich Solovyev
}

Yurii Petrovich Solovyev was born on 8 October 1944 in Krasnoyarsk, a city in south central Siberia, on the Yenisei river. In 1948, his family moved to Bryansk, a city in Russia which is 369 kilometers southwest of Moscow. He did his primary and secondary school education there. In 1963, he graduated with distinction from Bryansk Technical College of Mechanical Engineering. After three years of service in the Soviet Army, he entered the Mechanics and Mathematics Department of the Moscow State University in 1968. From that point on, his life was closely tied to the university. In 1973, he graduated from the Department of Mechanics and Mathematics with distinction. His diploma thesis [1] was published two years later while he was already working on his Candidate of Sciences thesis. In 1976, he defended this thesis and received his Candidate of Science degree, which in the Soviet Union was the equivalent of a Ph.D. degree. After that he became an assistant professor in the Department and from 1983 till 1992 he was a senior researcher. In 1988, he defended his Doctoral thesis and received his Doctor of Science degree. In the Soviet Union this degree was much higher than the Candidate of Science degree and relatively few mathematicians received it. In 1992, he was appointed Professor at the Department of Mechanics and Mathematics.

The early research papers of Solovyev including his diploma thesis reflected the interests of his supervisor A. S. Mishchenko. At that time these interests centered around topological methods in the theory of elliptic operators. One of the important problems here was to find an explicit elliptic operator on a smooth manifold which is equivariant, up to compact operators, under the action of an infinite discrete group and has nontrivial index. All known examples concerned only finite groups. Finiteness guaranteed that the universal cover is compact and therefore the classical Atiyah-Singer theory could be applied. On the other hand when the discrete group is infinite the universal cover is not compact and therefore the classical AtiyahSinger theory does not apply. Moreover there was even a problem to define properly the index of an elliptic operator.

Solovyev solved the problem above by constructing geometrically an explicit differential elliptic operator on $\mathbb{R}^{2}$ which commuted with the action of the lattice $\mathbb{Z}^{2}$ up to compact operators and gave a nontrivial element in $K$-homology $K_{0}\left(T^{2}\right)$ of the 2-torus. From this it follows immediately that for any orientable surface $S_{g}$ of genus $g$, every vector bundle over $S_{g}$ can be obtained from some Fredholm representation of a fundamental group $\pi_{1}\left(S_{g}\right)$. Combining this with previous results of Mishchenko one obtained a simple proof of Novikov's conjecture for fundamental groups of surfaces. 
Shortly thereafter he extended the approach above to cover much more complicated spaces, namely Bruhat-Tits buildings. These spaces were very new at that time. From this result it followed, in particular, that Novikov's conjecture held for a fairly broad class of discrete groups, namely for lattices in linear algebraic groups over locally compact local fields. This result is so strong that there has been in the meantime only slight improvements of it.

Next he discovered that higher signatures can be defined not only for smooth but also for rational homology manifolds. The discovery is based on the analysis of the way Hermitian $K$-theory is constructed using algebraic Poincare complexes because they generalize simultaneously Poincare duality on one hand and Hermitian quadratic forms on the other. Together with Mishchenko he constructed a theory of sheaves of algebraic Poincare complexes, and this led to a purely homotopical proof of Novikov's conjecture for those cohomology classes which are realized by Fredholm representations, and also to the derivation of Hirzebruch type formulae for arbitrary $C^{*}$-algebras.

These results made up his Candidate of Science thesis "Infinite dimensional representations of discrete groups and higher signatures".

Another problem that Solovyev solved using algebraic Poincaré complexes was a description of the subgroup in $L$-theory, a subdivision of Hermitian $K$-theory, consisting of all elements which are realized by closed combinatorial manifolds. He gave a description of such elements in purely homotopical terms (1981).

Hermitian $K$-theory played a role in the thesis and became the focus of Solovyev's work in the decade following his thesis. At first he defined higher Hermitian $K$-groups, applying Quillen's plus construction to the stable unitary groups of Bak, as Karoubi had also done. The resulting theory was called higher Hermitian $K$-theory. He then constructed Volodin Hermitian $K$-groups and showed they agreed with those obtained above by the plus construction. Since classical groups of types $C$ and $D$ are special cases of Bak's unitary groups, the result above shows that the plus and Volodin construction of symplectic (type $C$ ) $K$-theory agree and that the plus and Volodin construction of quadratic (type $D$ ) $K$-theory agree. On several occasions he conjectured that a similar result should hold for $K$-theory based on classical groups of type $B$. Next Solovyev turned his attention to the $\mathrm{K}$-theory of spaces. He began by designing a Volodin space for defining the $K-$ groups of spaces and showed that it was homotopically equivalent to the original construction of Waldhausen. This work was followed, of course, by making the (two) analogous constructions for the Hermitian $K$-theory of spaces and showing they were also homotopically equivalent.

Solovyev became interested next in dihedral (co)homology and its relation to Hermitian $\mathrm{K}$-theory. His interest in this topic was sparked by the development 
of cyclic (co)homology and its relation to the usual algebraic $K$-theory. He felt it was necessary to understand in a general way the notion of cyclic object and undertook this investigation together with his students R.L.Krasauskas, S.V. Lapin, and V.A.Kolosov. In a key paper of Krasauskas, the notion of an object with internal symmetries, which is a generalization of the notion of cyclic object, is described in two equivalent ways. One is called a category of type $\Delta$ and the other a skew simplicial group. It is shown that the category of such gadgets has a final object and this object has precisely 7 subobjects including itself and the trivial subobject. Moreover each of these objects defines a (co)homology theory. One of the subobjects is the so-called cyclic skew simplicial group and another the dihedral skew simplicial group. The reader should beware that a skew simplicial group is not a simplicial group. The cyclic subobject gives rise to cyclic (co)homology theory and the dyhedral one to dyhedral (co)homology theory. Solovyev together with his students investigated the latter theory for both rings with involution and spaces and established maps from the Hermitian $K$-theory of rings with involution to the dyhedral homology of rings with involution and from the Hermitian $K$-theory of spaces to the dyhedral homology of spaces. The last map led to an important isomorphism

$$
\pi_{n}\left({ }^{\varepsilon} K U(X)\right) \otimes \mathbb{Q} \equiv H D_{n}(\Omega X)
$$

where $K U(X)$ denotes the Hermitian $K$-theory space of a simply connected topological space $X$. This result is the Hermitian analog of the isomorphism $\pi_{n}\left(K_{i}(X)\right) \otimes \mathbb{Q} \equiv H C_{n}(X)$ of Burghelia where $K(X)$ denotes the Waldhausen $K$-theory space of $X$.

Dihedral homology was applied by Solovyov to compute invariants of the space of homotopy equivalences and invariants of the space of homeomorphisms of a combinatorial manifold. He showed that computing the rational homotopy groups of the homeomorphism group of a simply connected topological space can be reduced to computing the dihedral homology of certain objects which, in turn, can be computed by the minimal model method of Sullivan. These results made it possible to compute the rational homotopy groups of the homeomorphism groups of complex and quaternion projective spaces of certain Grassmannian manifolds.

His results of 1976-1987 were included in his Doctoral dissertation, "Unitary algebraic $K$-theory and its topological applications".

After obtaining his doctoral degree, Solovyev continued working on Hermitian $K$-theory, but his main interests switched to theoretical physics. In a long series of papers with V. V. Belokurov and E. T. Shavgulidze he developed a general approach for constructing absolutely convergent series for approximating functional integrals with arbitrary accuracy.

In the early 90 -s Solovyev created a special course on the mathematical 
foundations of quantum field theory. This course has now been taught for more than ten years in the Mechanics and Mathematics Department of Moscow State University.

Solovyev was a member of the editorial boards of several scientific journals. A journal called "Kvant", which was devoted to mathematics and physics for secondary school students, held an important place in his life. He was deputy editor of the journal from 1981 until 1994, and a member of the editorial board until the end of his life. During these years he largely determined the image and style of the journal and took part on a regular bases in selecting and editing papers. Possessing unquestionable literary talent and broad historical and general scientific erudition, Solovyev wrote a series of wonderful articles for "Kvant". His articles "van Roomen's challenge", "Evariste Galois", "Nikolai Ivanovich Lobachevskii", "Creators of the new astronomy", and others became real masterpieces in the popular scientific literature. His articles combined in an excellent linguistic style, scientific depth with clarity and precision.

Solovyev was interested in popular science and in the scientific motivation and scientific education of secondary school students. Solovyev also taught for several years at the Physics-Mathematical School No. 18. This famous school was founded by A.N. Kolmogorov in order to provide a very high level secondary school education, with emphasis in physics and mathematics, to exceptionally talented secondary school students selected from all over the Soviet Union. In 1987 he took part in the preparation and training of secondary school students to participate in the XXVIII International Mathematical Olympiad. In that year, the team of the Soviet Union won 3 gold and 3 silver medals. These activities were supplemented over many years by his popular science lectures at sessions of the Moscow Mathematical Society.

He always followed the latest scientific developments, and was up to date with the most important results not only in geometry and topology, but also in other areas. Apparently, he was one of the few who studied in detail the deduction of Fermat's last theorem from the Taniyama-Weil conjecture (and there were many more such examples).

Solovyev paid a lot of attention to his work with his students and to teaching at the Mechanics and Mathematics Department. Under his supervision 16 Candidate of Science thesis and 2 doctoral dissertations were defended. He taught every year basic and specialized courses in differential geometry and topology. He was a coauthor of a book of problems in geometry which was first published in 1978, and he later worked hard on preparing a substantially revised and augmented 4th edition which appeared in 2004 under the title "A collection of problems in geometry and topology". He led several seminars over his career. In recent years he took 
such an active part in organizing one of the oldest seminars in the Mechanics and Mathematics Department, the seminar of M. M. Postnikov in algebraic topology, that he became its full-fledged co-leader. For a long time Solovyev was a member of the executive committee of the Moscow Mathematical Society.

Yurii Petrovich Solovyev died suddenly and unexpectedly on September 11, 2003. He will forever remain in our memory and in the memory of his friends, colleagues, and students. We shall remember him as an enthusiastic researcher with a broad spectrum of interests, as a kind yet extremely demanding lecturer, and as a person of exceptional sensitivity who possessed an amazing gift for communication. The untimely departure of Yurii Petrovich is an irreplaceable loss to all who knew him.
A. S. Mishchenko
Th. Yu. Popelensky
E. V. Troitsky

\section{SELECTED PUBLICATIONS}

1. A theorem of Atiyah-Hirzebruch type for infinite-dimensional discrete groups, Vestnik Moskov. Univ. Ser. I Mat. Mekh. 1975, no. 4, 26-35; English transl., Moscow Univ. Math. Bull. 30:3/4 (1975), 77-85

2. Discrete subgroups, Bruhat-Tits buildings, and homotopy invariance of higher signatures, Uspekhi Mat. Nauk 35:1 (1975), 261-262 (Russian)

3. Homotopy invariants of rational homology manifold, Dokl. Akad. Nauk SSSR 230 (1976), 41-43; English transl., Soviet Math. Dokl. 17 (1976), 1260-1263

4. Problems in geometry, Izdat. Moskov. Gos. Univ., Moscow 1976 (with A. S. Mishchenko, S. P. Novikov, and A. T. Fomenko) (Russian)

5. Representations of $C^{*}$-algebras and signature formulae, Proc. 7th All-Union Topology Conf. (Minsk, 1977), Inst. Mat., Akad. Nauk Belorussk. SSR, Minsk 1977 (with A.S. Mishchenko) (Russian)

6. Homotopy invariance of higher signatures for discrete subgroups of algebraic groups, Proc. 7th All-Union Topology Conf. (Minsk, 1977), Inst. Mat., Akad. Nauk Belorussk. SSR, Minsk 1977 (Russian)

7. On infinite-dimensional representations of fundamental groups and formulae of Hirzebruch type, Dokl. Akad. Nauk SSSR 234 (1977), 761-764 (with A.S. Mishchenko); English transl., Soviet Math. Dokl. 18 (1977), 767-771

8. Classifying space for Hermitian K-theory, Trudy Sem. Vektor. Tenzor. Anal. 18 (1978), 140-168 (with A.S. Mishchenko); English transl., Selecta Math. Sov. 8:2 (1989), 159187 
9. Quillen constructions in Hermitian K-theory, Dokl. Akad. Nauk SSSR 253 (1980), 301-304; English transl., Soviet Math. Dokl. 22 (1980), 96-99

10. Representations of Banach algebras and formulae of Hirzebruch type, Mat. Sb. Ill (1980), 209-226 (with A.S. Mishchenko); English transl., Math. USSR-Sb. 39 (1981), 189-205

11. Signature realizable subgroups of the Wall group, Uspekhi Mat. Nauk 36:3 (1981), 223-224; English transl., Russian Math. Surveys 36:3 (1981), 266-267

12. Collection of problems on differential geometry and topology, Izdat. Moskov. Gos. Univ., Moscow 1981 (with A.S. Mishchenko and A.T. Fomenko) (Russian)

13. Differential geometry, Izdat. Moskov. Gos. Univ., Moscow 1981 (with A.M. Vasil'ev) (Russian)

14. Topology, Izdat. Moskov. Gos. Univ., Moscow 1982 (with Yu. G. Borisovich and V. G. Zvyagin) (Russian)

15. Homotopy multiplication in the representing space of Hermitian K-theory, Dokl. Akad. Nauk SSSR 258 (1982), 30-34 (with A.I. Nemytov); English transl., Soviet Math. Dokl. 23 (1982), 479-482

16. BN-pairs and Hermitian K-theory, Algebra. Collection Dedicated to the 90th Anniversary of the Birth of O. Yu. Schmidt, Izdat. Moskov. Gos. Univ., Moscow 1982, pp. 102-118 (with A.I. Nemytov) (Russian)

17. The equivalence of two definitions of algebraic $K$-theory of spaces, Vestnik Moskov. Univ. Ser. I Mat. Mekh. 1982, no. 6, 8-12; English transl., Moscow Univ. Math. Bull. 37:6 (1982), 5-9

18. Hermitian K-theory of topological spaces, Proc. Internat. Topology Conf. (Leningrad, 1982), Nauka, Leningrad 1983, pp. 100-112 (Russian)

19. Geometric structures on the moduli space of gauge fields with interaction. Differential Geometry and Global Analysis, no. 12, Voronezh 1984 (Russian)

20. Problems in differential geometry and topology, Mir, Moscow 1985 (with A. S. Mishchenko and A.T. Fomenko)

21. Dihedral homology and Hermitian K-theory of topological spaces, Uspekhi Mat. Nauk 41:2 (1986), 195-196 (with R. L. Krasauskas); English transl., Russian Math. Surveys 41:2 (1986), 203-204

22. Characteristic classes in algebraic K-theory, Vestnik Moskov. Univ. Ser. I Mat. Mekh. 1986, no. 1, 75-76 (with Yu.A. Zhuraev and A.S. Mishchenko); English transl., Moscow Univ. Math. Bull 41:1 (1986), 80-82

23. Algebraic K-theory of quadratic forms, Itogi Nauki i Tekhniki: Algebra, Topologiya, Geometriya, vol. 24, VINITI, Moscow 1986, pp. 121-194; English transl., J. Soviet Math. 44 (1989), 319-371 
24. Dihedral homology and cohomology, Vestnik Moskov. Univ. Ser. I Mat. Mekh. 1987, no. 4, 28-32 (with R. L. Krasauskas and S. V. Lapin); English transl., Moscow Univ. Math. Bull. 42:4 (1987), 36-40

25. Dihedral homology and cohomology. Basic concepts and constructions, Mat. Sb. 133 (1987), 25-48 (with R. L. Krasauskas and S.V. Lapin); English transl., Math. USSR-Sb. 61 (1988), 23-47

26. Differential geometry, 2nd rev. aug. ed., Izdat. Moskov. Gos. Univ., Moscow 1988 (with A.M. Vasil'ev) (Russian)

27. Rational Hermitian K-theory and dihedral homology, Izv. Akad. Nauk SSSR. Ser. Mat. 52 (1988), 935-969 (with R. L. Krasauskas); English transl., Math. USSR-Izv. 33 (1989), 261-293

28. Topology, Izdat. Moskov. Gos. Univ., Moscow 1988 (with B. A. Dubrovin) (Russian)

29. Rational homotopy type of Hermitian K-theory, Vestnik Moskov. Univ. Ser. I Mat. Mekh. 1990, no. 5, 77-80 (with V. A. Kolosov); English transl., Moscow Univ. Math. Bull. $45: 5$ (1990), 62-64

30. Topology of four-dimensional manifolds, Uspekhi Mat. Nauk 46:2 (1991), 145-202; English transl., Russian Math. Surveys 46:2 (1991), 167-232

31. Differential geometry, Izdat. Serbsk. Akad. Nauk, Belgrad 1991 (with Ch. Dolichanin) (Russian)

32. Analysis on manifolds, Izdat. Serbsk. Akad. Nauk 1991 (with Ch. Dolichanin) (Russian)

33. Algebraic K-theory and dihedral homology, Humboldt-Universitat, Berlin 1992

34. Lectures on homology with internal symmetries, Internat. Center for Theoret. Physics, Trieste 1992

35. Elliptic functions and elliptic curves, Izdat. Nezavisim. Univ., Moscow 1993 (with V. V. Prasolov) (Russian)

36. Symmetric bar-construction and combinatorial topological models, Vestnik Moskov. Univ. Ser. I Mat. Mekh. 1994, no. 3, 90-92 (with V. A. Kolosov); English transl., Moscow Univ. Math. Bull. 49:3 (1994), 56-58

37. Algebraic equations and theta-functions, Izdat. Nezavisim. Univ., Moscow 1994 (with V. V. Prasolov) (Russian)

38. Geometric methods in electrodynamics, Izdat. Serbsk. Akad. Nauk, Belgrad 1994 (with Ch. Dolichanin) (Russian)

39. On computation of Hochschild and cyclic homology of homogeneous spaces, Int. J. Shape Model. 1:2 (1984), 119-139

40. New perturbation theory for quantum field theory: convergent series instead of asymptotic expansions, Modern Phys. Lett. A 10 (1995), 3033-3041 
41. $C^{*}$-algebras and elliptic operators in the topology of manifolds, Faktorial, Moscow 1996 (with E. V. Troitsky) (Russian)

42. Method of approximate computation of path integrals, using perturbation theory with convergent series. I, Teoret. Mat. Fiz. 109 (1996), 51-59 (with V. V. Belokurov and E. T. Shavgulidze); English transl., Theoret. and Math. Phys. 109 (1996), 1287-1293

43. Method of approximate computation of path integrals, using perturbation theory with convergent series. II, Teoret. Mat. Fiz. 109 (1996), 60-69 (with V. V. Belokurov and E. T. Shavgulidze); English transl., Theoret. and Math. Phys. 109 (1996), 1294-1301

44. Elements of the mathematical apparatus of quantum field theory, Lecture Notes on Theoretical and Mathematical Physics, vol. 1, Kazan’ 1996 (with V. V. Belokurov) (Russian)

45. V. S. Yarunin and M. A. Smondyrev (ed.), Convergent series perturbation theory for functional integrals, Proc. Internat. Conf. on Path Integrals (Dubna, 1996), Joint Inst. Nuclear Res., Dubna 1996, pp. 122-126 (with V. V. Belokurov and E. T. Shavgulidze)

46. On computation of Feynman integrals with large coupling constant, Modern Phys. Lett. A 12 (1997), 661-672 (with V. V. Belokurov and E.T. Shavgulidze)

47. Elliptic functions and elliptic integrals, Amer. Math. Soc., Providence, RI 1997 (with V. V. Prasolov)

48. Perturbation theory with convergent series for functional integrals with respect to the Feynman measure, Uspekhi Mat. Nauk 52:2 (1997), 153-154 (with V. V, Belokurov and E. T. Shavgulidze); English transl., Russian Math. Surveys 52 (1997), 392-393

49. Elliptic functions and algebraic equations, Faktorial, Moscow 1997 (with V. V. Prasolov) (Russian)

50. Computation of functional integrals using convergent series, Fundam. i Prikl. Mat. 3 (1997), 693-713 (with V. V. Belokurov and E. T. Shavgulidze) (Russian)

51. Rational points on elliptic curves, Sorosovskii Obrazovatel'nyi Zhurnal (Soros Educational J.) 1997, no. 10 (Russian)

52. Accumulation of structural radiation defects in quartz in cooling systems: basics for dating, Phys. Chern. Minerals 25:2 (1998), 243-248 (with D. G. Koshchug)

53. A summation method for divergent series, Uspekhi Mat. Nauk 54:3 (1990), 153-154 (with V. V. Belokurov and E. T. Shavgulidze); English transl., Russian Math. Surveys 54 (1999), 626-627

54. A method of summing divergent series to any accuracy, Mat. Zametki 68:1 (2000), 24-3b (with V. V. Belokurov and E.T. Shavgulidze); English transl., Math. Notes 68 (2000), 22-31

55. Perturbation theory with convergent series for computing physical quantities given by finitely many terms of a divergent series in the traditional perturbation theory, Teoret. Mat. Fiz. 123 (2000), 452-461 (with V.V. Belokurov and E. T. Shavgulidze); English transl., Theoret. and Math. Phys. 123 (2000), 792-800 
56. ESR dating of cooling natural systems, Vestnik Moskov. Univ. Ser. IV Geolog. 1999, no. 4,31-39 (with D.G. Koshchug); English transl., Moscow Univ. Geolog. Bull. 54:4 (1999), 28-42

57. Thermal stability of paramagnetic centers in quartz in natural environments, Proc. 9th Internat. Conf. on Luminescence and Electron Spin Resonance Dating, Rome, 1999 (with D.G. Koshchug)

58. Problems in algebra and number theory, Izdat. Moskov. Gos. Univ., A.N. Kolmogorov School, Moscow 1999 (Russian)

59. A general approach to the calculation of functional integrals and the summation of divergent series, Fundam. i Prikl. Mat. 5 (1999), 363-376 (with V.V. Belokurov and E.T. Shavgulidze) (Russian)

60. $C^{*}$-algebras and elliptic operators in differential topology, Amer. Math. Soc., Providence, RI 2001 (with E.V. Troitsky)

61. New perturbation theory for quantum field theory: convergent series instead of asymptotic expansions, Acta Appl. Math. 68:1-3 (2001), 71-104 (with V.V. Belokurov and E.T. Shavgulidze)

62. Computation of the $\beta$-function in the $\phi^{4}$-model in a broad interval of values of the coupling constant, Vestnik Moskov. Univ. Ser. Ill Fiz. Astron. 2001, no. 1, 3-9 (with V.V. Belokurov, E.T. Shavgulidze, and I. L. Yudin); English transl., Moscow Univ. Phys. Bull. 56:1 (2001), 1-6

63. Examples of computations in the framework of a new perturbation theory with convergent series, Vestnik Moskov. Univ. Ser. III Fiz. Astron. 2001, no. 2, 23-27 (with V.V. Belokurov, E.T. Shavgulidze, and I. L. Yudin); English transl., Moscow Univ. Phys. Bull. 56:2 (2001), 23-30

64. Elliptic curves and modern algorithms of number theory, Institute of Computer Research, Moscow-Izhevsk 2003 (with V. A. Sadovnichii, E.T. Shavgulidze, and V.V. Belokurov) (Russian)

65. Lie-Cartan pairs and characteristic classes in noncommutative geometry, Contemporary Geometry and Related Topics, World Scientific, Singapore 2004, pp. 351-374 (with T. Yu. Popelensky)

66. Collection of problems in differential geometry and topology, 4th ed., Fizmatlit, Moscow 2004 (with A.S. Mishchenko and A.T. Fomenko) (Russian)

67. The existence of functional integrals in a model of quantum field theory on a loop space, Uspekhi Mat. Nauk 59:5 (2004), 163-164 (with V.V. Belokurov and E.T. Shavgulidze); English transl., Russian Math. Surveys 59 (2004), 982-983 
A. S. MishCHENKo asmish@higeom.math.msu.su

Dept. of Mechanics and Mathematics

Moscow State University

Moscow, 119992

Russia

TH. YU. POPELENSKY

popelens@mail.ru

Dept. of Mechanics and Mathematics

Moscow State University

Moscow, 119992

Russia

E. V. TROITSKY

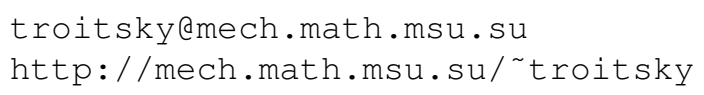

Dept. of Mech. and Math.

Moscow State University

119992 GSP-2 Moscow

Russia 